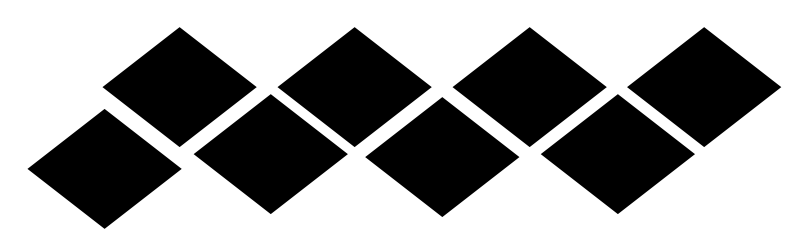

\title{
Le cadre spatio-temporel dans "À quoi rêvent les loups" de: Yasmina Khadra
}

\section{Mustafa Jabir Ahmed}

$$
\begin{aligned}
& \text { مجلة كلية الاداب - جامعة جنوب الوادي - العدد (10) لسنة • ب • بم }
\end{aligned}
$$

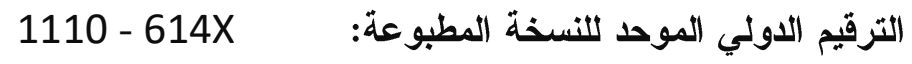

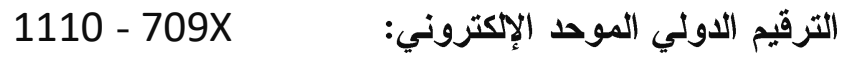

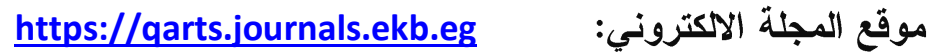




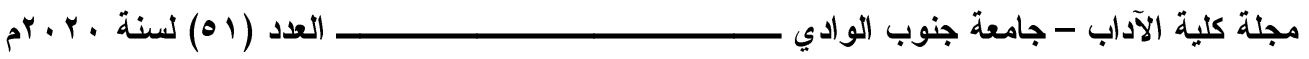 \\ Résumé}

Yasmina Khadra, comme il était un des écrivains de la littérature d'urgence, a centralisé son roman sur les événements de la violence faits par la mouvance terroriste en Algérie à la fin du $\mathrm{XX}^{\mathrm{e}}$ siècle. « $\grave{A}$ quoi rêvent les loups » lance un cri douloureux contre la violence et l'injustice sociale en Algérie.

Le roman moderne fond sur plusieurs éléments dont le temps et l'espace sont les plus efficaces. À ce titre, le temps et l'espace sont des principes fondamentaux de ce roman qui nous permet de mettre en lumière les faits historiques et en même temps d'incarner la vie sociopolitique des Algériens pendant cette période critique de son histoire. «À quoi rêvent les loups» est donc une cohésion formidable de la fiction et de la réalité dans un cadre sociopolitique bien exposé.

Mots clés: Khadra, temps, espace 


\section{I- La théorie du temps :}

Le temps est le composant le plus important dans le roman. Il est le moteur qui dirige le véhicule par lequel nous donnons aux lieux et aux actions une signification. Aucun de temps, c'est-à-dire qu'il n'y a aucune d'actions et les personnages seraient sans doute immobiles. Par le temps, nous pouvons constamment transformer aux lecteurs l'histoire passée et la vision vers le futur. Tout comme il existe au cinéma, il est donc possible de jouer avec le temps dans le récit. Nous pouvons l'accélérer, le ralentir, retourner en arrière, etc. Nous pouvons également choisir le type du roman et les héros, c'est-à-dire, le narrateur de l'histoire. Au contraire d'autres arts ( la peinture et la sculpture) qui portent sur l'espace, le roman est en général une production complètement temporelle qui se distingue par sa dimension historique, sa relation de la vie, de la réalité et des événements historiques. René Wellek et Austin Warren montrent que:

"Il faut en général ranger la littérature dans la catégorie des arts inscrits dans le temps (...). Dans beaucoup de grands romans, les hommes naissent, grandissent et meurent; les personnages se développent, se modifient; on voit parfois même changer une société tout entière " ${ }^{1}$

L'importance fonctionelle du temps paraît évidemment dans ses participations définitives à déterminer le genre et l'orientation thématique des récits. Nous pouvons caractériser, à travers le temps, le roman historique et celui des science-fictions, le roman d'aventure et celui romantique. Nous pouvons différencier aussi des genres romanesques orientés vers le passé ou le futur, vers le réel ou la fiction. Le roman est donc soumis d'une grande partie à l'axe temporel sur lequel nous pouvons construire un cadre vivant de nos souvenirs

1 WELLEK René \& WARREN Austin, La théorie littéraire, Traduit de l'anglais par AUDIGIER Jean-pierre \& GATTÉGNO Jean, Paris, Seuil, 1977, p. 301 
d'enfance, faire écho d'un passé que nous ne voyons jamais la profendeur de ces échos, ni les traces qu'il s'est passé.

Bien entendu, l'auteur doit soigneusement choisir le temps dans lequel il présente son roman. Le bon choix du temps d'écriture contribue à la dramatisation des récits. Nous pouvons considérer le temps comme l'une de ces formes qui établissent essentiellement l'univers fictif. Il s'agit que le temps dans un roman est un temps faux dès que nous entrons dans cet univers jusqu'à la fin du roman. L'auteur doit avoir une grande mentalité, une vaste imagination en vue de persuader le lecteur que les actions du récit sont communément réelles. Il utilise des moyens différents et des références temporelles plus effectives pour nous donner l'illusion du réel.

"Le mot irrite encore parfois le romancier sérieux, qui sait fort bien que la fiction est moins étrange et plus représentative que la vérité (...). La réalité d'une œuvre de fiction - c'est-à-dire l'illusion de réalité qu'elle crée, l'effet produit sur le lecteur qu'il s'agit là d'une traduction convaincante de la vie- n'est ni nécessairement ni avant tout un réalisme des circonstances, du détail, du banal $»^{2}$

En effet, nous pouvons constater qu'il y a différence entre le récit et le roman. Au sujet du récit, il représente un temps complètement imaginaire, un peu touché du réel, mais aussi il est raconté d'une manière plus différente que le roman en donnant la parole aux animaux et aux monstres de l'espace extérieur par exemple. Au contraire, le roman est assez proche de notre vie et plus touché du réel. Il semble comme l'intermédiaire par lequel nous pouvons voir de nos yeux, écouter de nos oreilles nos cries, nos réactions, les moments joyeux ou les plus tristes, la vie et la mort. Le roman est donc réaliste et le récit est mythique comme les distingue René Wellek :

${ }^{2}$ WELLEK René \& WARREN Austin, La théorie littéraire, op.cit., p. 299. 
"Le Roman (novel) est un tableau de la vie et des mœurs réelles, et de l'époque au cours de laquelle il est écrit. Le récit romanesque (romance) écrit dans une langue noble et solennelle, décrit des choses qui ne sont jamais arrivées et qui n'ont guère de chance d'arriver. " ${ }^{3}$

Pour approfondir de plus en plus, nous faisons une étude longue et détailleuse sur le thème du récit et du roman. En ce qui concerne le récit, il nous montre donc, sous une forme imaginaire, des actions qui ne se sont pas passées que dans nos croyances et nos imaginations. Il nous expose, d'une manière assez différente mais plutôt fictive, notre crainte intérieure, notre contemplation universaire.Tout ça ne prend pas sa signification qu'à l'intérieur du récit. Michel Butor nous indique que:

"Le récit nous donne le monde, mais il nous donne fatalement un monde faux. Nous sommes à chaque instant obligés de faire intervenir dans les récits une distinction entre le réel et l'imagination, frontière très poreuse, très instable. " ${ }^{4}$

Il est en effet évident qu'un événement dans le récit ne peut pas se raconter qu'à partir des instruments narratifs variés qui sont certes le narrateur, l'intrigue et les images fictives. La fiction, selon cette perspective, fait constamment réunir à la fois les actions du réel et de l'imagination. C'est possible donc de savoir qu'un récit peut contenir une sorte de l'imagination; mais il est très difficile de distinguer entre les actions de la vérité et la fiction dans le même récit.

«Nous savons bien que dans ce qu'on nous raconte, il y a des choses qui ne sont pas vraies, non seulement

3 Ibid., p.302

${ }^{4}$ BUTOR Michel, Essais sur le roman, Paris, Gallimard, 1969, pp. 110-111 
des erreurs mais des fictions(...).Le seul moyen de dire la vérité, c'est de confronter inlassablement, méthodiquement, ce que nous racontons d'habitude avec ce que nous voyons, entendons, avec les informations que nous recevons, c'est donc "travailler" sur le récit »

À propos du roman, il ne contient que des modèles fictifs de la temporalité, ou d'autre sens, le temps dans le roman est un temps faux en utilisant des techniques narratives différentes et complexes (des retours en arrière, des anticipations ....etc). Il est possible donc de faire des retours en arrière, des prophéties dans le futur et même aussi de sauter des bouts d'histoire. En effet, les critiques nous montrent qu'il y a deux types du temps: d'un côté le temps de la fiction (le temps raconté) et de l'autre côté, le temps de la narration ( Le temps racontant). Nous allons passer en revue ces deux types dans les pages suivantes.

Nous pouvons bien remarquer qu'il y a dans le roman trois temps différents. Nous pouvons distinguer entre le temps de l'aventure, le temps de l'écriture et le temps de la lecture. Butor nous a proposé ces trois temps:

"Dès que nous abordons la région du roman, il faut superposer au moins trois temps: celui de l'aventure, celui de l'écriture, celui de la lecture " ${ }^{6}$

Le temps de l'aventure, il représente la période de l'histoire. Ce temps fait une relation étroite entre les actions actuelles avec son passé et ses conséquences à l'avenir, mais il ne peut pas respecter la linéarité des événements. Il y a alors une impossibilité de raconter son histoire selon un ordre purement chronologique. D'après ce point de vue, le roman est un tissu complexe des événements temporels qui se traduit

${ }^{5}$ Ibid., p. 111. 
par des anticipations et des retours en arrières au cours de l'histoire. Jean Bessière nous montre:

"Toute représentation est à la fois son actualité et son passé, et histoire de cette double temporalité. Le passé se trouve entièrement dans le présent; cette coalescence apparaît comme le gage de l'avenir de représentation ${ }^{7}$

Le temps de l'écriture représente le moment où l'écrivain décide de commencer l'écriture. Il réfère aussi à la durée de composer l'œuvre. La nuance que l'écrivain met en exergue au moment de l'écriture est de plus en plus importante que l'ordre chronologique. À ce titre, il utilise des techniques romanesques variées. Roland Barhes dit:

"La technique romanesque, elle-même indissocialbe du moment de l'écriture, car l'écrivain, alors même qu'il emprunte ou les refuse, est tributaire des modes et des procédés de son époque. "8

La composition du roman est plus importante, d'ailleurs la méthode de présentation doit fournir au roman son rythme propre. Composer un roman doit être différent de la production d'une pièce en concernant l'atmosphère, des descriptions, du choix du narrateur et des temps, l'analyse des sentiments et l'incarnation minutieuse des actions afin de faire donner au roman sa forme audiovisuelle. Le roman doit se dérouler harmonieusement. Il doit mettre en compte les moyens narratifs attractifs loin de la rupture. En concernant ce sujet, Goldenstein commente en disant:

"Le scripteur recourt à la dramatisation pour marquer un moment important du récit, une

7 BESSIÈRE Jean, L'Ordre du descriptif, Paris, Presses universitaires, 1988, p. 97.

8 BARHES Roland, Le degré zéro de l'écriture, Paris, Seuil, 1953, p. 20. 
évolution décisive de l'action. Un roman policier ou d'aventures rebondira de scène en scène avec un minimum de liens descriptifs $" 9$

Quant au temps de la lecture, il est bien touché aux lecteurs, leurs orientations et les différences individuelles entre eux. En outre, le roman doit être, en partie inséparable, lié à son époque, c'est-à-dire que le roman doit être convenu de temps en temps à l'évolution des pensées des lecteurs. Le roman prend son importance quand le lecteur goûte plus particulièrement les événements par lesquels il peut faire une comparaison entre le temps de la lecture et la durée de ce qu'on lit. L'un des signes les plus nécessaires dans chaque roman est l'adaptation littéraire entre la durée de la lecture et la société à laquelle on s'adresse le roman. Le roman doit ainsi stimuler les lecteurs et leur donner l'envie de lire en choisissant des sujets qui possèdent des rapports à leur vie actuelle. Youssef Abouali évoque ce point de vue en disant :

"Le roman est un discours qui a été figé dans le temps. Cela ne veut aucunement dire qu'il est fermé sur lui-même ou bien encore qu'il ne communique pas/plus avec l'extérieur. Tout au contraire, le texte romanesque est extrêmement bavard et ses lectures ne sont en fait que des tentatives de le faire taire. "10

Un autre point de vue qui montre que le roman doit soumettre à l'ordre narratif. Nous ne concernons pas ici l'ordre chronologique des événements qui correspond à sa linéarité dans un roman, mais plutôt, il s'agit de la succession des événements, d'une manière harmonieuse et attractive, s'appuyant sur des techniques narratives variées qui se caractérisent d'une part par va-et-vient entre le présent et le passé et

${ }^{9}$ GOLDENSTEIN Jean-Pierre, Lire le roman, Belgique, Boeck université, 2005, p. 45.

10 ABOUALI Youssef, Yasmina Khadra ou la recherche de la vérité : Étude de la trilogie sur le malentendu entre l'Orient et l'Occident, Paris, L'Harmattan, 2013, p. 336. 
d'autre part par la vitesse des actions. (l'accélération et le ralentissement). Goldenstein nous indique que :

"Les procédés techniques utilisés par le scripteur relèvent des choix esthétiques historiquement datés, des conventions plus ou moins implicites, fondatrices d'un pacte de production et de consommation narrative dans une société donnée. ${ }^{11}$

\section{II- Le temps racontant et le temps raconté :}

Chaque roman s'est raconté ou bien s'est déroulé dans un temps déterminé. Le temps y représente ainsi une notion principale à respecter, non seulement parcequ'il réunit les événements produits dans un temps déterminé, mais aussi car la narration d'un roman a besoin de classifier les événements sur un axe temporel. Cette temporalité et le choix du temps convenable ont alors pour l'objet d'assurer la cohérence entre tous les éléments du roman d'un côté et d'éviter l'ennui et le mal compris chez le lecteur de l'autre côté.

En effet, nous pouvons distinguer une double référence de temporalité dans tous les récits. L'une est le temps de l'histoire qui se réfère au déroulement des événements selon sa linéarité (Le temps raconté), l'autre est le temps de la narration qui concerne le rythme et la manière de raconter ou d'exposer les actions (Le temps racontant).

"Le récit est une séquence deux fois temporelle.(...)

et que les théoriciens allemands désignent par l'opposition entre erzählteZeit (temps de l'histoire) et Erzählzeit (temps du récit)» ${ }^{12}$

${ }^{11}$ GOLDENSTEIN Jean-Pierre, Lire le roman, op.cit., p. 46.

${ }^{12}$ GENETTE Gérard, Figures III, Discours du récit, Paris, Le Seuil, 1972, p. 77. 


\section{A- Le temps raconté ( le temps de l'histoire) :}

Bien entendu, le temps dans le roman est un temps fictif dès que nous commençons à lire le roman jusqu'à la fin. C'est un temps descriptif imaginaire de tout ce que le narrateur nous raconte dans le récit. Il nous expose cependant une description plus détaillée de tout ce qu'il voit afin de mener le lecteur à rendre interactif, à entrer vivement dans son univers. C'est bien sûr le temps de la chose racontée. Il est évident que ce temps apparaît largement dans le roman de la récitation épique et les bandes dessinées qui dépendent la succession des événements, selon la succession des actions ou la succession des images.

Ce genre de raconter porte sur une temporalité relative fournie par la succession des événements dans un ordre presque chronologique. Les pages et les chapitres se succèdent. Le monde du roman est un monde fictif qui se forme progressivement avec les éléments du roman, ses décors, ses personnages et sa linéarité. Cet univers temporel apparaît clairement dans les indications temporelles qu'il évoque en profitant des heures, des jours et des années. L'ordre temporel de succession des événements dans la diégèse ${ }^{13}$ correspond sans aucun doute à la durée des actions dans le temps réel. Genette dit:

"Le livre est un peu plus tenu par la fameuse linéarité du signifiant linguistique.(...) et le temps qu'il faut pour le consommer est celui qu'il faut pour le parcourir ou le traverser, comme une route ou un champ ${ }^{14}$

Le temps que nous pouvons désigner ici dans le roman, peut relier étroitement à l'analyse fictive des actions narratives d'après sa linéarité régulière de raconter. Il s'agit alors la succession chronologique où

${ }^{13}$ Ce terme est cité par Gérard Genette dans son livre : Figure III ( p. 72). II signifie l'univers du roman, il est aussi égale à l'histoire du récit.

${ }^{14}$ GENETTE Gérard, Figures III, Discours du récit, op.cit., p. 78. 
chaque événement possède à la fois une relation positive avec son précédant et son suivant. L'histoire est donc une interprétation du réel. Nous concernons ici l'analyse des activités humaines observées par les yeux qui se déroulent dans la vie quotidienne comme la description des lieux et des personnages. Ce temps peut évoquer une heure, une journée ou bien il peut s'étendre pour des jours, des mois, ou d'une longue période d'années qui vont pour comprendre des générations.

"Le temps de la fiction, ou temps raconté, représente la durée du déroulement de l'action. Facteur déterminant, il permet la transformation des situations narratives et des personnages qui leur procurent un soutien figuratif. " ${ }^{15}$

L'enchaînement chronologique apparaît particulièrement dans le roman picaresque "où la succession chronologique est tout " ${ }^{16}$. Dans le roman des aventures, les faits se déroulent successivement selon l'ordre historique des actions. Le temps de l'histoire se réfère donc au temps vécu par les personnages de l'histoire. Le temps indique ainsi des répères qui sont reliés entre eux, qui contribuent largement à classifier le roman dans des pages et des chapitres en vue de mettre les événements dans l'ordre où ils doivent historiquement se passer. Le roman peut donc organiser autour d'un système chronologique comme des jalons qui se succèdent naturellement. Michel Butor indique :

"Chaque événement va être, l'origine d'une enquête sur ce qui l'a précédé et ce qui l'a suivi, va, peut le suivre, de proche en proche ${ }^{17}$

\section{B- Le temps racontant ( le temps de la narration ) :}

${ }^{15}$ GOLDENSTEIN Jean-Pierre, Lire le roman, op.cit., p. 125.

16 WELLEK René \& WARREN Austin, La théorie littéraire, op.cit., p. 301.

17 BUTOR Michel, Essais sur le roman, op.cit., p. 117. 
Il est impossible de raconter tous les événements du roman dans une succession linéaire unique, c'est pourquoi le romancier recourt parfois à utiliser des techniques plus efficaces en vue de réaliser la combinaison entre les éléments de l'intrigue dans le roman. Selon ce point de vue, l'écriture littéraire ne peut pas avancer toujours de manière chronologique dans le même sens que le temps de l'histoire utilise.

Le romancier, en écrivant un roman, vise à captiver l'attention du lecteur, à éviter l'ennui probablement produit par la succession des éléments de la narration selon sa linéarité chronologique. Il est obligé parfois de raconter les événements dans un ordre varié selon le moment de les rapporter. Le temps de la narration est donc le rythme que le narrateur utilise pour raconter les actions du roman. Il présente des jeux temporels variés qui correspondent au genre du roman ou au personnage héros. Nous pouvons ainsi trouver que le héros une fois se souvient au passé et dans une autre fois, il plonge dans les rêves à l'avenir. Goldenstein nous répond à l'importance du temps racontant:

"Pourquoi les romanciers ne se bornent-ils pas à
raconter tout simplement les événements dans
l'ordre chronologique de leur succession? Ce
bouleversement du temps de la narration permet de
manifester la durée. Le roman, par cet artifice,
devient une prise de conscience de la durée. "

De fait, le temps racontant représente un axe essentiel dans la narration du roman. Il rend en compte une représentation clairement différente à mesure de leur déroulement dans la vie quotidienne. Le narrateur recourt parfois à un genre d'accélération ou de ralentissement pour régler la durée des actions qui ne s'enchaînent pas linéarement selon l'ordre de leur succession; mais vivement selon les sautes de la

${ }^{18}$ GOLDENSTEIN Jean-Pierre, Lire le roman, op.cit., p. 130. 
mémoire. Nous pouvons remarquer certains mots qui peuvent présenter de longues périodes de plusieurs années. Au contraire, un récit complet peut raconter une histoire de quelques jours.

"Tel roman de cinq cents pages traite d'une journée dans la vie d'un héros tout comme tel autre mince récit rapporte l'existence entière d'un personnage. $~^{19}$

Le temps racontant est donc le temps fondamental du roman. Or, ce temps est exposé d'une manière spécifique en utilisant plusieurs temps grammaticaux envisagés soit vers le passé, soit vers le présent ou le futur. Ces temps grammaticaux portent sur l'action qui se déroule au passé accompli ou inaccompli. David Fontaine nous expose le temps de la narration et ses quatre composants selon la position de l'action que le narrateur raconte dans le roman notamment en concernant la relation de l'avant et de l'après:

\section{B-1- La narration ultérieure:}

Il s'agit de raconter les événements qui se sont déroulés au passé en utilisant l'imparfait, le passé simple et le passé composé. Le narrateur, à l'impossibilité de raconter tous les événements en même temps, recourt parfois à utiliser la narration ultérieure afin de montrer plusieurs temps pour raconter l'histoire dans l'ordre qu'il souhaite d'achever.

"La narration ultérieure est de loin la plus courante. L'histoire est racontée après coup et d'une coulée, instantanément pour ainsi dire. ${ }^{20}$

Le narrateur emploie donc les différents temps du passé pour exposer une forme accomplie ou inaccomplie à l'action. Décrire une action au passé d'une manière particulière, en utilisant l'imparfait, le

19 Ibid., p. 129.

20 FONTAINE David, La poétique: introduction à la théorie générale des formes littéraires, Paris, Nathan, 1993, p.52. 
passé simple et le passé composé ne donnent pas le même sens à cette action. Le passé simple, par ses caractéristiques, est le temps des actions achevées. Il permet alors la raconter d'une manière naturelle et claire, des actions qui peuvent se succéder les unes après les autres. Selon Roland Barthes:

"Le passé simple, pierre d'angle du Récit, signale toujours un art. Par son passé simple, le verbe fait implicitement partie d'une chaîne causale, il participe à un ensemble d'actions solidaires et dirigées, il fonctionne comme le signe algébrique d'une intention. ${ }^{21}$

L'emploi du passé simple dans le roman permet également de mettre en évidence la réalité. Ce temps contribue, de façon cohérente, à mettre les événements en ordre pour rendre le lecteur mieux comprendre du déroulement des actions. C'est pourquoi le passé simple est le moyen idéal d'un narrateur en écrivant une narration bien élaborée, bien comprise, mais aussi plus attractive. Le passé simple est donc très utile dans le roman parce que, grâce à lui, le verbe exprime un acte clos, défini et vivant. Ce temps est proprement le mécanisme du rythme dans le roman, parce qu'il a l'habilité d'introduire ou bien de cacher amplement les faits irréels dans le monde visible de la réalité. Barthes ajoute que:

"Le passé simple est donc finalement l'expression d'un ordre, et par conséquent d'une euphorie. Grâce à lui, la réalité n'est ni mystérieuse, ni absurde; elle est claire, presque familière, à chaque moment rassemblée et contenue dans la main d'un créateur ${ }^{22}$ 


\section{B-2- La narration antérieure :}

Dans ce genre de la narration, le récit fait des sautes vers l'avenir. Le narrateur utilise alors le futur en racontant les actions et les faits imaginés par les personnages, qui ne se sont pas encore réalisés. Nous le trouvons jouer un rôle déterminant dans l'intrigue en présentant un conseil ou des précautions. En effet, ce type de la narration est assez rare et inscrit généralement en un seul paragraphe dans le roman. Il ne relève que d'une forme des prévisions (prophétie ou rêve).

"Trouver fréquement des anticipations est plus rare parce que projeter son image dans l'avenir et se voir dans telle ou telle situation de façon concrète et détaillée n'est pas propre à tout le monde. ${ }^{23}$

\section{B-3- La narration simultanée :}

Le narrateur raconte les actions courantes et actuelles. Il se trouve au moment même où les événements se déroulent. Il utilise alors le présent pour présenter et exposer, d'une manière directe, les actions contemporaines qu'il vit.

"La narration simultanée est strictement contemporaine de l'histoire qu'elle énonce, leur durée coïncident en tout point. ${ }^{24}$

\section{B-4- La narration intercalée :}

Nous pouvons remarquer que ce genre est le plus compliqué car il rend le roman instable surtout le roman de plusieurs héros. Dans ce

${ }^{23}$ ACLIMANDOS Myriam, Les structures du récit dans l'œuvre romanesque d'Adré Chedid: Le sixième jour, les marches de sable, Thèse de maîtrise, Faculté des lettres, Université du caire, 1991, p. 85.

24 FONTAINE David, La poétique: introduction à la théorie générale des formes littéraires, op.cit., p. 53. 
genre de narration, nous trouvons parfois deux héros dans le même récit. C'est pourquoi la narration intercalée, d'après ce cas, est très necessaire à faire l'équilibrage et à faire va-et-vient entre les deux personnages. Ce genre apparaît en particulier comme le montre Genette, dans le roman épistolaire à plusieurs destinataires et dans le journal intime en expliquant que :

"Le dernier type est a priori le plus complexe, puisqu'il s'agit d'une narration à plusieurs instances(....). Ici, le narrateur est tout à la fois encore le héros et déjà quelqu'un d'autre. ${ }^{25}$

\section{III- Les rapports temporels bi-axiaux ${ }^{26}$ :}

Bon écrivain est égal un bon roman. Le roman pour être bien élaboré, doit mettre en compte la coïncidence entre le temps racontant et le temps raconté. La relation harmonieuse entre les deux temps permet au lecteur de déterminer les objectifs, soit lointains soit plus proches, que l'écrivain a faits en produisant certains effets. Il est important alors de s'interroger sur les rapports qui réunissent le temps de l'histoire et celui de la narration. Ces rapports temporels permettent au romancier de constituer des jeux avec le temps. Il est bien connu que le narrateur doit nécessairement raconter le roman, quoi qu'il soit, aux temps différents (présent - passé - futur). La manière que le narrateur utilise à raconter le roman et le système de repérage temporel qu'il tente d'installer, sont des bases sur lesquelles nous pouvons juger le roman. Le récit comporte ainsi une double référence temporelle dont les rapports sont essentiels dans le texte littéraire et font partie du jeu narratif car le narrateur ne peut raconter les événements d'une manière inséparable. Il récourt donc à raconter les actions dans le désordre.

${ }^{25}$ GENETTE Gérard, Figures III, Discours du récit, op.cit., pp. 229-230.

${ }^{26}$ II s'agit les rapports du double axe entre le temps de l'histoire et le temps de la narration. Ce terme est tiré de l'expression "Schémas bi-axiaux» cité par Goldenstein dans son livre «Lire le roman », p.131. 
Nous pouvons distinguer trois types de relations entre le temps de l'histoire et celui de la narration selon: l'ordre, la durée et la fréquence.

Goldenstein nous introduit, de façon explicite, cette relation qui réunit les deux temps dans le même cadre littéraire. Ces deux temps sont étroitement liés dans les actions de l'intrigue, notamment en concernant l'ordre de la succession. Dans son livre "Lire le roman", il nous donne des réponses définitives à la question du choix des unités temporelles en présentant alors des schémas bi-axiaux entre les événements.

"Le temps de la fiction et de la narration sont unis par une série de rapports que l'on peut représenter graphiquement pour la simplification de l'expose ${ }^{27}$

Goldenstein présente largement les trois relations entre les deux temps. Ici, nous passons en revue les rapports de l'ordre, Le genre le plus courant. Dans la figure suivante, nous trouvons que le roman s'est présenté en deux axes principaux dont l'un est le temps de la fiction $(\mathbf{F})$ et l'autre axe est celui de la narration $(\mathbf{N})$. Les unités du temps sont classifiés sur les deux axes comme ( $\left.a / a^{\prime}-b / b^{\prime}-c / c^{\prime}\right)$ où (a/ a') réfère au temps contemporain. Les événements sont, alors, racontées au présent. Nous pouvons l'appeler “ le degré zéro". (b/ b') représente un retour en arrière. Le narrateur utilise le temps passé à raconter. (c/ c') représente les rêves et l'anticipation. Dans ce cas, Le narrateur utilise le future pour raconter les actions de l'avenir.

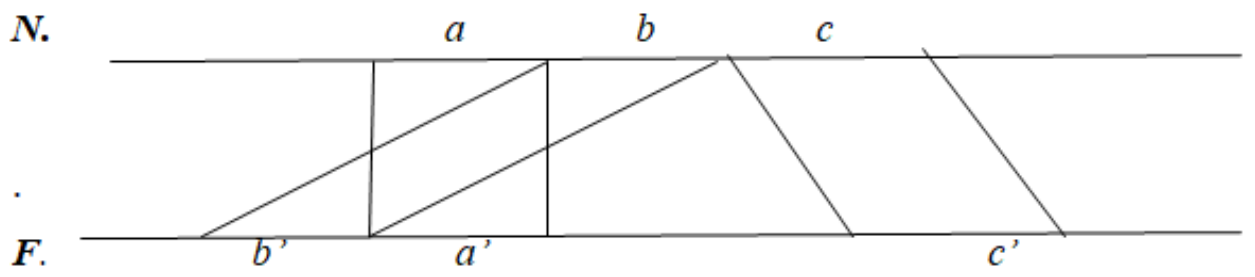

(Figure 1) ${ }^{(28)}$

27 GOLDENSTEIN Jean-Pierre, Lire le roman, op.cit., p. 131.

${ }^{28}$ Ibidem. 
Selon cette figure, nous pouvons remarquer que l'ordre des unités sur les deux axes ne correspond pas l'un à l'autre. Sur l'axe de la fiction, les événements se classifient selon son ordre chronologique. Au contraire, L'autre axe de la narration nous présente les actions comme le narrateur les racontent dans le roman.

Une autre figure, Goldenstein nous présente, concerne le cas d'équilibrage entre le temps racontant et celui raconté. Il s'agit le moment du dialogue entre les personnages du récit où nous pouvons lire la parole que se déroule entre les personnages en prenant le même temps de prononcer le dialogue au style direct. Dans ce cas de la proportion, nous pouvons remarquer que l'unité (a) sur l'axe narratif correspond à celle ressemblabe sur l'axe fictif $(\mathbf{N}=\mathbf{F})$.

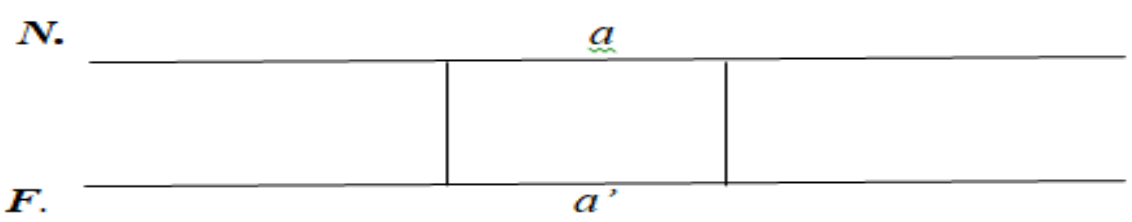

\section{(Figure 2) ${ }^{29}$}

La dernière figure, que nous présentons, concerne le cas de la narration intercalée. Dans ce type de la narration, le roman devient instable car il met l'accent, tantôt sur l'histoire racontée, tantôt sur la narration. Nous voyons, selon cette figure, que le narrateur raconte les actions de plusieurs personnes dans le même temps du déroulement des faits. Il nous propose encore ce qui survient tout à coup en différentes situations au même moment. Ce genre est largement utilisé dans la description ( $\mathbf{N} / / \mathbf{F})$.

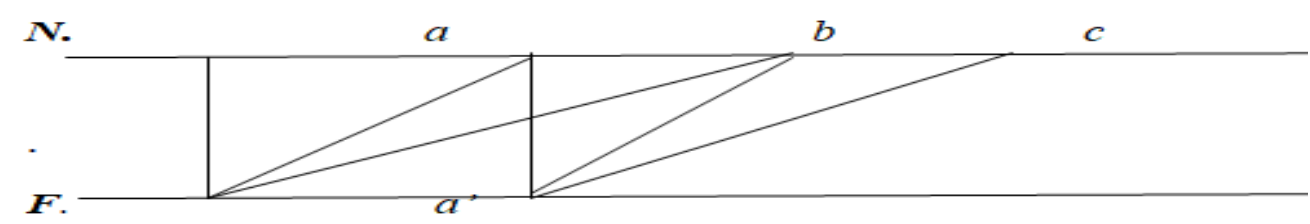

(Figure 3) ${ }^{30}$

${ }^{29}$ Ibid., p. 133. 


\section{Conculision :}

Le temps est un élément fondamental dans la production du roman. Chaque événement doit se dérouler dans un temps précis. Ces événements sont présentés dans le récit sous deux types de raconter, dont l'un concerne l'histoire fictive caractérisée par sa linéarité chronologique où les actions se succèdent selon les jalons temporels, l'autre type concerne la manière de la proportion narrative du récit raconté caractérisé par la complexité temporelle. En racontant un roman, C'est très difficile à raconter tous les événements selon l'ordre chronologique. Il ya toujours des ruptures. Ces ruptures apparaissent, largement dans le retour en arrière comme le flash-back, et rarement dans l'anticipation.

"À quoi rêvent les loups" avec ses trois grandes parties précédées par une terrible prologue, met l'accent sur la situation d'Algérie pendant la décennie noire à la fin $\mathrm{du} \mathrm{xx}^{\mathrm{e}}$ siècle. La misère du peuple de ce pays qui a subi quotidiennement la violence et la mort sous les mains des intégristes terroristes. Khadra a utilisé plusieurs techniques narratives en vue de tirer l'attention de ses lecteurs. On le trouve accélérer le ryhme puis tente de le relntir pour présenter une longue description et dans certains cas, il laisse les personnages du roman se dialoguer. Khadra est un grand talent moderne en maîtrisant le temps.

Le romancier utilise sa grande habilité pour maîtriser l'intrigue romanesque. Il joue bien avec le temps, en utilisant le passé, le présent et le futur, afin de persuader le lecteur de la réalité des événements. Il est clair que le roman est généralement raconté dans le temps passé, et de façon particulière dans le passé simple. Le romancier faire intervaler les temps pour tirer l'attention de ses lecteurs à lire le roman.

${ }^{30}$ Ibid., p. 138. 


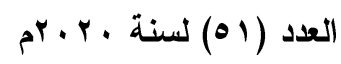
مجلة كلية الآداب - جامعة جنوب الوادي

\section{Bibliographie}

* Le corpus:

1- KHADRA Yasmina, À quoi rêvent les loups, Paris, Éd.Julliard, 1999.

* Ouvrages consacrés à l'auteur de l'étude

1- ABOUALI Youssef, Yasmina Khadra ou la recherche de la vérité : Étude de la trilogie sur le malentendu entre l'Orient et l'Occident, Paris, L'Harmattan, 2013.

\section{* Ouvrages généraux :}

1- BARHES Roland, Le degré zéro de l'écriture, Paris, Seuil, 1953.

2- BESSIÈRE Jean, L'Ordre du descriptif, Paris, Presses universitaires, 1988.

3- BUTOR Michel, Essais sur le roman, Paris, Gallimard, 1969.

4- FONTAINE David, La poétique: introduction à la théorie générale des formes littéraires, Paris, Nathan, 1993.

5- GENETTE Gérard, Figures III, Discours du récit, Paris, Le Seuil, 1972.

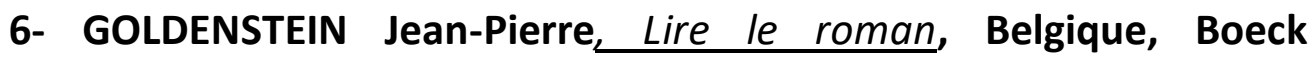
université, 2005.

7- WELLEK René \& WARREN Austin, La théorie littéraire, Traduit de l'anglais par AUDIGIER Jean-pierre \& GATTÉGNO Jean, Paris, Seuil, 1977.

\section{* Mémoires et Thèses :}

1- ACLIMANDOS Myriam, Les structures du récit dans l'œuvre romanesque d'Adré Chedid: Le sixième jour, les marches de sable, Thèse de maîtrise, Faculté des lettres, Université du caire, 1991. 
Le cadre spatio-temporel dans "À quoi rêvent les loups Mustafa Jabir

الإطار الزمني والمكانى فى رواية " بم تحلم الأنـاب " للكاتب ياسمينا خضرا

$$
\text { مصطفى جابر أحمد آدم }
$$

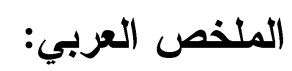

باعتباره أحد كتاب أدب الطوارئ، فقد قام الكاتب الجزائرى ياسمينا خضر ا بتسليط الضوء فى روايته على أحداث العنف التى شهدتها البلاد على أيدى الجماعات الإرهابية

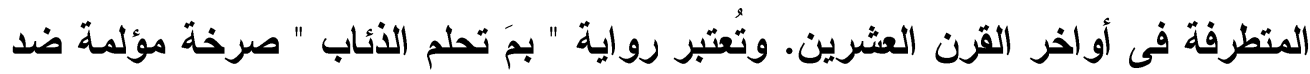
العنف والظلم الاجتماعي الطبقى داخل الجزائر.

إنّّ الرواية الحديثة ترتكز على عدة عناصر أساسية والتى يعتبر عنصرى" الزمان

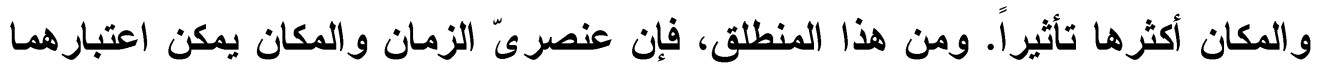
ركائز أساسية لهذه الرواية فهى تسلط الضوء على الأحداث التاريخية وفى الوقت عينه، تجسد الحياة الاجتماعية والسياسية داخل المجتمع الجزائرى خلا هذه الحقبة التاريخية الحرجة. وبالتالي فإن رواية "بمَ تحلم الأنئب" تعرض مزيج متناست من الحقيقة والخيال وذلك فى إطار اجتماعي وسياسى · 